\title{
Change in Lymphocyte Subsets of Holstein Calves Caused by Short-term Transportation
}

\author{
Nobue KANEMATSU ${ }^{1 *}$, Reiji AOKI ${ }^{2}$, Hiroyuki SHINGU ${ }^{1}$, \\ Tsutomu ASADA ${ }^{3}$, Naoko MORIYA ${ }^{2}$, Yosuke KOBAYASHI', \\ Fumihiro OHTANI ${ }^{1}$ and Madoka SUTOH ${ }^{4}$ \\ ${ }^{1}$ Division of Animal Metabolism and Nutrition, Institute of Livestock and Grassland Science, NARO \\ (Tsukuba, Ibaraki 305-0901, Japan) \\ ${ }^{2}$ Division of Animal Products Research, Institute of Livestock and Grassland science, NARO (Tsukuba, \\ Ibaraki 305-0901, Japan) \\ ${ }^{3}$ Gunma Prefectural Livestock Experiment Station (Maebashi, Gunma 371-0103, Japan) \\ ${ }^{4}$ Division of Animal Environment and Waste Management Research, Institute of Livestock and \\ Grassland science, NARO (Tsukuba, Ibaraki 305-0901, Japan)
}

\begin{abstract}
In order to study the possibility of using lymphocyte subsets as a stress indicator, the changes of the subset proportion in peripheral blood were examined after transportation. Six 3-month-old male Holstein calves were transported for about $3 \mathrm{~h}$ in a lorry via an ordinary road. Blood samples were collected at the expected unloading time on the day before transport (T0), immediately after unloading (T1), $24 \mathrm{~h}$ after unloading (T2), and 1 week after unloading (T3). CD2, CD4, CD8, CD21, CD25, WC1, and NKp46 antigens on peripheral blood mononuclear cells were measured by flow cytometry. There was an increase in the proportions of $\mathrm{CD} 25+$ cells and $\mathrm{WC} 1+\gamma \delta \mathrm{T}$ cells at $\mathrm{T} 1$ and $\mathrm{T} 2$. The proportions of NK cells increased at T1, including an increase in the population of CD2- NK cells. CD2+ NK cells did not show any change. The proportions at $\mathrm{T} 1$ were lower than at $\mathrm{T} 3$ in $\mathrm{CD} 21+\mathrm{B}$ cells. CD4+ and $\mathrm{CD} 8+\alpha \beta \mathrm{T}$ cells did not change significantly, although $\mathrm{CD} 2+\mathrm{T}$ cells showed a trend toward reduction at $\mathrm{T} 1$. Consequently, $\mathrm{CD} 2-\mathrm{NK}$ cells and $\mathrm{WC} 1+\gamma \delta \mathrm{T}$ cells might offer potential as stress index for calf transport.
\end{abstract}

Discipline: Animal industry

Additional key words: $\gamma \delta \mathrm{T}$ cell, NK cell, transport stress, cattle

\section{Introduction}

The concept of animal welfare for farm animals in Japan remains in the initial stages, but is slowly becoming popular among consumers. Although a practice guideline to be followed by farmers exists (Japan Live Stock Technology Association 2011), there is further need for scientific methods of assessing the condition of animals. Cattle experience various kinds of stress, but transport is considered one of the most stressful events. Transport is known to affect the immune system of cattle via many factors (Swanson \& Morrow-Tesch 2001, Knowles \& Warriss 2007). There are various immunological or hormonal indices for a study on stress, although a perfect index does not exist. For example, the level of cortisol in the blood has been recognized as a representative indicator of stress, including the elevation caused by acute stress as well as the low level resulting from chronic stress (Christison \& Johnson 1972). However, the acute stress-related increase often masks other stressors and confuses the effect, since the increase begins within a few minutes and rapidly reaches a peak. Therefore, novel indices have always been required for a study.

Lymphocyte subpopulation analysis is a newer immunological index for stress in cattle (Van Kampen \& Mallard 1997, Anderson et al. 1999, Fell et al. 1999). This analysis may possibly express the effect from chronic environmental stress in addition to that from acute stress. Nevertheless, only a few studies have examined transport stress (Riondato et al. 2008, Ishizaki \& Kariya 2010). Long-distance transport has increased in Japan, although short distance transport is more common in many areas. Young cattle have traditionally been entrusted to public farms in each area, so

*Corresponding author: e-mail kaja@affrc.go.jp

Received 11 August 2015; accepted 2 June 2016. 
as to reduce the feed cost and each farm household's work. Recently, some prefectures have also begun to recommend intensive grazing for the effective utilization of abandoned farmland. The aim of this study was the verification and development of a study on lymphocyte subsets for few hours of transport.

\section{Materials and Methods}

This study was carried out in strict accordance with the guidelines approved by the Animal Care and Use Committee of the National Institute of Livestock and Grassland Science (Permit Number: 10032415). All efforts were made to minimize suffering.

Six 3-month-old male Holstein calves, each weighing approximately $100 \mathrm{~kg}$, had been raised in group feeding. Standard formula feed and hay had been given to the calves, because their rumen develops to the same level as that of adult cattle at three months after birth. The calves were individually tied in a covered 8-ton lorry $(>6.2 \times 2.1 \mathrm{~m})$ without bedding, and transported via an ordinary road. The journey from the farm (in Maebashi) to the National Institute of Livestock and Grassland Science (in Tsukuba) took about $3 \mathrm{~h}(110 \mathrm{~km})$. While traveling, the calves were able to lie or stand. On the day before transport, blood samples were taken by external jugular venepuncture at the same time as the expected arrival time on the next day (10:00; T0). Upon arrival, samples were collected immediately after unloading (10:00; T1), $24 \mathrm{~h}$ after unloading (T2), and 1 week after unloading (T3). The animals developed no clinical abnormalities during the experiment. After unloading, the calves were housed in individual indoor stalls that enable direct contact between the animals.

Peripheral blood mononuclear cells (PBMC) were isolated using Lymphoprep (Axis-Shield Poc AS). The labeled antibodies were commercialized mouse monoclonal antibodies conjugated to phycoerythrin (PE), fluorescein isothiocyanate (FITC), or Alexa Fluor 647 (AbD Serotec). Table 1 shows the specificities and clones of the antibodies used for the flowcytometric phenotyping of lymphocyte subpopulations. About $100 \mu \mathrm{L}$ of $1.0 \times 10^{6}$ separated PBMC from each sample were incubated for $20 \mathrm{~min}$ with monoclonal antibodies at $4^{\circ} \mathrm{C}$ in the dark. Cells were then washed and resuspended in $1 \mathrm{~mL}$ of PBS. A minimum of 50000 events from a gated lymphocyte population were acquired and analyzed using a Gallios Flowcytometer (Beckman Coulter Inc.). FlowJo software (Tree Star Inc.) was used for data analyses based on the cell surface marker expression.

Plasma was separated using a refrigerated centrifugation $\left(2200 \times \mathrm{g}\right.$ for $30 \mathrm{~min}$ at $\left.4^{\circ} \mathrm{C}\right)$ and then stored at $-30^{\circ} \mathrm{C}$. Cortisol concentration was measured using a commercial EIA kit with a QUARTUS ImmunoCube Auto-Analyser
(Kainos Inc.).

Analysis of variance was performed using a repeatedmeasures mixed linear model (MIXED procedure) of SAS Add-In 6.1 for Microsoft Office (SAS Institute Japan Ltd.) in order to compare temporal variation. Day was included as a fixed effect and individual calves as a random effect in the model. Multiple comparisons for each effect were controlled for using the Tukey method and $P<0.05$ was considered significant.

\section{Results}

The counts of PBMC were constant $\left(2-5 \times 10^{6} / \mathrm{ml}\right.$ whole blood) and did not change significantly in any of the animals. Alterations in the percentage of lymphocyte subsets expressing the selected differentiation antigens are shown in Fig. 1. The proportion of $\mathrm{CD} 2+$ cells in PBMC was the highest at all points in this study (Fig. 1A). It showed a trend toward reduction at T1 $(P=0.06)$, as compared with at $\mathrm{T} 0$. The proportions of $\mathrm{CD} 21+\mathrm{B}$ cells increased at $\mathrm{T} 3$, as compared with at $\mathrm{T} 1 . \mathrm{CD} 4+$ and $\mathrm{CD} 8+$ $\alpha \beta \mathrm{T}$ cells, did not change significantly.

The proportion of CD25+ cells was increased at $\mathrm{T} 1$ and remained significantly high at $\mathrm{T} 2$, as was the case for $\mathrm{WC} 1+\gamma \delta \mathrm{T}$ cells (Fig. $1 \mathrm{~B}$ ). In contrast to $\mathrm{CD} 4+$ or $\mathrm{CD} 8+$ $\alpha \beta T$ cells (including the $\alpha \beta$ chain in the receptor), $\gamma \delta \mathrm{T}$ cells have another type of receptor consisting of the $\gamma \delta$ chain. And most bovine $\gamma \delta \mathrm{T}$ cells have a surface molecule stainable by the WC1 antibody (Pollock \& Welsh 2002). The proportion of $\mathrm{WC} 1+\gamma \delta \mathrm{T}$ cells increased significantly at $\mathrm{T} 1$, and at $\mathrm{T} 3$ returned to the same level as at T0. There was a significant increase in the total proportion of NK cells between $\mathrm{T} 0$ and $\mathrm{T} 1$. The proportion of CD2- NK cells also increased significantly at T1 (Table 2). CD2- NK cells at $\mathrm{T} 3$ were lower than that at $\mathrm{T} 1$. The proportion of $\mathrm{CD} 2+\mathrm{NK}$ cells did not show any change.

Plasma cortisol concentration showed a significant increase at T1, and returned to the T0 level at T2 (Table 3). The value at $\mathrm{T} 3$ was significantly lower than that at $\mathrm{T} 0$.

\section{Discussion}

The transport of live cattle is known to be stressful, and can lead to immune suppression, particularly in calves (Swanson \& Morrow-Tesch 2001). Meanwhile, it is not yet clear whether changes in the relative proportions of lymphocyte subsets in cattle follow a circadian rhythm, as cortisol does (Riondato et al. 2008). Therefore, prior to transport, the first sample was collected at the same time of day as the expected arrival time after transport on the following day.

It has been reported that short-term exposure to acute psychological stress results in an increase in $\gamma \delta \mathrm{T}$ cells in 
Table 1. Explanation of antibodies

\begin{tabular}{llll}
\hline \hline Specificity & Clone & Fluor & Cellular expression \\
\hline CD2 & CC42 & FITC & $\alpha \beta$ T cell, $\gamma \delta$ T cell*, NK cell \\
CD4 & CC8 & FITC & $\alpha \beta$ T cell \\
CD8 & CC63 & Alexa Fluor 647 & $\alpha \beta$ T cell \\
CD21 & CC21 & PE & B cell \\
CD25 & ILA111 & PE & B, T, NK cell \\
WC1 & CC15 & FITC & $\gamma \delta$ T cell \\
NKp46 & AKS1 & PE & NK cell \\
\hline
\end{tabular}

The specificities and clones of antibodies used for the flowcytometric phenotyping of lymphocyte subpopulations (PE: phycoerythrin, FITC: fluorescein isothiocyanate), and the objective cells. * low ratio.
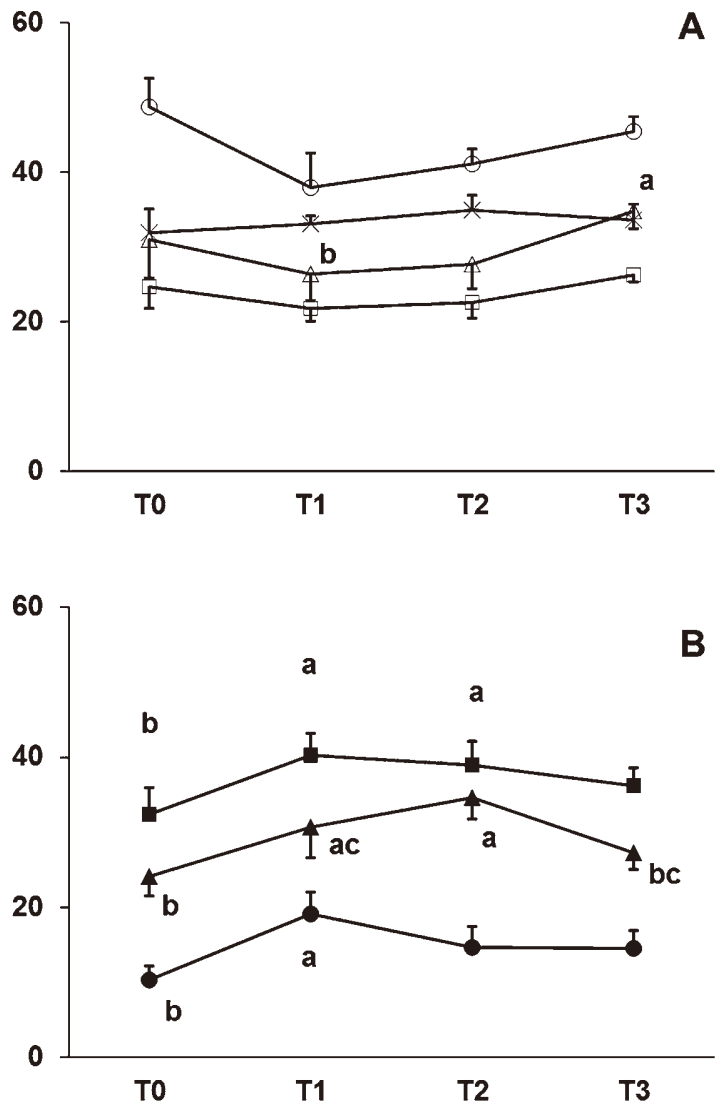

Fig. 1. Proportion of lymphocyte subset in PBMC.

The percentage of circulating lymphocyte subsets in the peripheral blood mononuclear cells (PBMC) of calves before departure (the same time as the expected arrival time on the next day (10:00; T0)), immediately after unloading (T1), $24 \mathrm{~h}$ after unloading (T2) and 1 week after unloading (T3). Values are presented as mean \pm SE ( $\mathrm{n}=$ 6). The subsets are shown as cells that are positive for an antibody to A. CD2 (open circle), CD8 (cross), CD21 (open triangle), or CD4 (open square); B. CD25 (closed square), WC1 (closed triangle), or NKp46 (closed circle) antigens. Different subscript letters indicate significant differences in the population $(\mathrm{P}<0.05)$. humans (Anane et al. 2010), although in calves, a decrease after $14 \mathrm{~h}$ of transport was shown by Riondato et al. (2008). The opposite result observed after $3 \mathrm{~h}$ of transport in this study may be due to different durations of transport, and the intensity of stress. The significantly high levels of $\mathrm{WC1}+$ $\gamma \delta \mathrm{T}$ cells remained evident the next day. Baldwin et al. (2000) reported a significant fluctuation in the proportion of $\gamma \delta \mathrm{T}$ cells coinciding with an environmental change. In this study, calves were not only transported but also moved to a different farm. $\gamma \delta \mathrm{T}$ cells are the first line of immunity in young animals, similar to NK cells (Pollock \& Welsh 2002). It is possible that a novel environment, possibly including exposure to new microbes, affected increases in the $\mathrm{WC1}+$ $\gamma \delta \mathrm{T}$ cell population at $\mathrm{T} 2$.

Because NK cells have low percentage of white blood cells (Graham 2009), detecting the clear variation caused by stress was considered difficult. Therefore, we used PBMC, excluding granulocytes occupying the greater part of the white blood cell population. The significant increase in the proportion of NK cells was evident at T1. It has been reported that the number of circulating NK cells peaked shortly after $2 \mathrm{~h}$ of transport (Ishizaki \& Kariya 2010), suggesting that a few hours of transport can result in an increase in NK cells. The CD2 molecule is the receptor for activation on both T cells and NK cells (McNerney \& Kumar 2006), although it is not expressed on most WC1+ $\gamma \delta$ T cells (Davis et al. 1996). Storset et al. (2004) reported that in cattle, most circulating NK cells are CD2+ and that CD2- cells comprise a low percentage. In this study, only the smaller CD2- NK cell population increased significantly just after transport, as did the total NK cell population. It has been reported that the CD2- subset shows signs of being more activated in circulation (Boysen et al. 2006). Thus, the CD2- subset may react rapidly and more clearly than the CD2+ subset (Riondato et al. 2008). Meanwhile, the proportion of CD2- NK cells in this study showed a decrease between $\mathrm{T} 1$ and $\mathrm{T} 3$. This may be due to change ahead of the 
Table 2. Proportion of NKp46+cells in PBMC

\begin{tabular}{lcccc}
\hline \hline & T0 & T1 & T2 & T3 \\
\hline Total NK & $10.3 \pm 1.8^{\mathrm{b}}$ & $19.1 \pm 2.8^{\mathrm{a}}$ & $14.7 \pm 2.8$ & $14.5 \pm 2.4$ \\
CD2+NK & $8.2 \pm 1.6$ & $11.9 \pm 2.8$ & $9.8 \pm 1.9$ & $11.0 \pm 2.1$ \\
CD2- NK & $2.1 \pm 0.2^{\mathrm{b}}$ & $7.2 \pm 1.0^{\mathrm{a}}$ & $4.9 \pm 1.0^{\mathrm{ab}}$ & $3.5 \pm 0.4^{\mathrm{bc}}$ \\
\hline
\end{tabular}

The percentage of total NK cells (detected with NKp46 antigen), CD2+NK cells, and CD2-NK cells in the peripheral blood mononuclear cells (PBMC) of calves before departure (T0), immediately after unloading (T1), $24 \mathrm{~h}$ after unloading (T2), and 1 week after unloading (T3). Data are presented as the means $\pm \mathrm{SE}(\mathrm{n}=6)$. Different subscript letters indicate significant differences in the population $(P<0.05)$.

Table 3. Changes in plasma cortisol

\begin{tabular}{cccc}
\hline \hline T0 & T1 & T2 & T3 \\
\hline $2.89 \pm 0.58^{\mathrm{b}}$ & $4.39 \pm 0.64^{\mathrm{a}}$ & $1.54 \pm 0.17^{\mathrm{bc}}$ & $0.85 \pm 0.32^{\mathrm{c}}$ \\
\hline
\end{tabular}

Changes in the concentration $(\mu \mathrm{g} / \mathrm{dL})$ of plasma cortisol $(\mathrm{n}=6)$ before departure (T0), immediately after unloading (T1), $24 \mathrm{~h}$ after unloading (T2), and 1 week after unloading (T3). Data are presented as the means \pm SE $(n=6)$. Different subscript letters indicate significant differences $(P<0.05)$.

total NK population, affected by a new environment. Then there is also the possibility that only CD2- NK cells are able to respond to such environmental stimuli sensitively, as both $\gamma \delta \mathrm{T}$ and NK cells play important roles in the innate immune system.

$\mathrm{CD} 25$, the $\alpha$-chain of the IL-2 receptor, is expressed on activated T-cells, B-cells, and NK cells (Waldmann et al. 1998). Thus, the increase in CD25+ cells observed in this study may have resulted from an increase in CD25 on $\mathrm{WC} 1+\gamma \delta \mathrm{T}$ cells and $\mathrm{NK}$ cells.

Long-term $(14 \mathrm{~h})$ transport decreased the proportion of CD4+ and CD8 $+\mathrm{T}$ cell just after arrival, although the decrease of the CD21+ B cell did not occur until the next day (Riondato et al. 2008). The results of the CD4+ T and CD8+ T cells did not show a significant change after transport in this study. Therefore, the $\alpha \beta T$ cell may not vary the proportion in a short time. In contrast, the CD21+ B cell at T3 was significantly higher than at T1. Short transport and the succeeding new environment may affect the B cell more clearly than the $\alpha \beta T$ cell, although the effect to the cell may also vary due to distance.

A positive correlation between circulating NK cell counts and plasma cortisol levels after transport has been reported (Ishizaki \& Kariya 2010). Although the relationship and the mechanism of action remain unclear, the reaction to stress in the lymphocyte subpopulation is thought to be slower than the rapid increase in cortisol. The stress may have already begun before loading, because the novelty of encountering unfamiliar humans and the behavior witnessed by calves for the first time are strong stressors for them (Grandin 2007). The increase in cortisol at $\mathrm{T} 1$ observed in the current study suggested that arrival time was when the animals were experiencing severe stress. However, a higher level at T0 than at T2 and T3 suggests that pre-transport stress may already have begun on the previous day. Novel human behavior and the first capture for collecting blood might affect the cortisol concentration at $\mathrm{T} 0$ in a sensitive calf, although other indices could not represent the results from an acute stressor until blood was collected. Then the cortisol levels had decreased significantly a week after arrival. Habituation due to our repeated handling and restraint for approximately 1 week might have been the cause of the decrease, functioning to lower the basal cortisol level (Grandin 2007). Furthermore, the decline and low level of cortisol could also have induced the decrease in CD2- NK cells at $\mathrm{T} 3$.

In summary, we substantiated that a few hours of transport can lead to an increase in bovine NK cells. In particular, CD2- NK cells might reflect the effect of transport on stress levels. Moreover, as well as NK cells, the study indicated the possibility that $\gamma \delta$ T cells could also be a useful index for short-term transport. It is still unknown how the lymphocyte population may change depending on stress from the breeding environment. To improve livestock welfare, further detailed studies on the associations between stress and the lymphocyte indices are required. 


\section{Acknowledgements}

The authors would like to thank Dr. T. Kokuho (National Institute of Animal Health, Tsukuba, Japan) and Dr. H. Ishizaki (National Institute of Livestock and Grassland Science, Nasushiobara, Japan) for their technical advice.

In this research work we used the supercomputer of AFFRIT, MAFF, Japan.

\section{References}

Anderson, B.H. et al. (1999) The Effect of Dexamethasone on Some Immunological Parameters in Cattle. Vet. Res. Commun. 23, 399-413.

Anane, L.H. et al. (2010) Phenotypic characterization of $\gamma \delta T$ cells mobilized in response to acute psychological stress. Brain Behav. Immun. 24, 608-614.

Baldwin, C.L. et al. (2000) Rapid changes occur in the percentage of circulating bovine $\mathrm{WC} 1+\gamma \delta \mathrm{Th} 1$ cells. Res. Vet. Sci. 69, 175-180.

Boysen, P. et al. (2006) Bovine CD2/NKp46+ cells are fully functional natural killer cells with a high activation status. BMC Immunol. 7, 10.

Christison, G.I. \& Johnson, H.D. (1972) Cortisol turnover in heat-stressed cows. J. Anim. Sci. 35, 1005-1010.

Davis, W.C. et al. (1996) Analysis of monoclonal antibodies specific for the gamma delta TcR. Vet. Immunol. Immunopathol. 52, 275-283.

Fell, L.R. et al. (1999) Associations between temperament, performance and immune function in cattle entering a commercial feedlot. Aust. J. Exp. Agr. 39, 795- 802.

Graham, E. M. et al. (2009) Natural killer cell number and phenotype in bovine peripheral blood is influenced by age. Vet. Immunol. Immunopathol. 132, 101-108.

Grandin, T. (2007) Behavioural Principles of Handling Cattle and Other Grazing Animals under Extensive Conditions. Livestock Handling and Transport. CABI Publishing, Wallingford, UK. 44-64.

Ishizaki, H. \& Kariya, Y. (2010) Road transportation stress promptly increases bovine peripheral blood absolute NK cell counts and cortisol levels. J. Vet. Med. Sci. 72, 747-753.

Knowles, T.G. \& Warriss, P.D. (2007) Stress physiology of animals during transport. Livestock Handling and Transport. CABI Publishing, Wallingford, UK. 312-328.

McNerney, M.E. \& Kumar, V. (2006) The CD2 family of natural killer cell receptors. Curr. Top. Microbiol. Immunol. 298, 91-120.

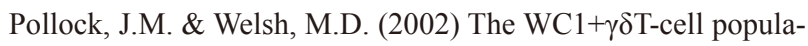
tion in cattle: a possible role in resistance to intracellular infection. Vet. Immunol. Immunopathol. 89,105-114.

Riondato, F. et al. (2008) Effects of road transportation on lymphocyte subsets in calves. Vet. J. 175, 364-368.

Storset, A.K. et al. (2004) NKp46 defines a subset of bovine leukocytes with natural killer cell characteristics. Eur. J. Immunol. 34, 669-676.

Swanson, J.C. \& Morrow-Tesch, J. (2001) Cattle transport: Historical, research, and future perspectives. J. Anim. Sci. 79, E102-E109.

Van Kampen, C. \& Mallard, B.A. (1997) Effects of peripartum stress and health on circulating bovine lymphocyte subsets. Vet. Immunol. Immunopathol. 59, 79-91.

Waldmann, T. et al. (1998) Interleukin-2, interleukin-15, and their receptors. Int. Rev. Immunol. 16, 205-226. 